\title{
Fundamentos de la filosofía hermenéutica: Heidegger y Gadamer
}

\section{ANTECEDENTES}

Antes de cristalizar como filosofía en Verdad y Método (1960) de Hans-Georg Gadamer, la hermenéutica tiene un largo recorrido vinculado a la interpretación de textos religiosos, literarios, históricos y jurídicos. En la primera mitad del siglo XIX, Friedrich Schleiermacher, a pesar de ser teólogo de profesión, elabora una teoría general de la comprensión independiente de la teología y de otros ámbitos específicos de aplicación. Inspirado en Schleiermacher, Guillermo Dilthey desarrolla, en el siglo siguiente, una hermenéutica filosófica, que se propone aportar el fundamento gnoseológico a las ciencias del espíritu, en tanto que temática y metódicamente independientes de las ciencias de la naturaleza. Pero es Martín Heidegger quien da el paso decisivo desde una hermenéutica que asume una tarea particular de la filosofía hacia una filosofía propiamente hermenéutica, al hacerse cargo del fenómeno de la comprensión como algo más que una forma de conocimiento o un sistema de reglas metodológicas, a saber como una determinación ontológica del hombre y un rasgo definitorio de la filosofía como tal en tanto que expresa la apertura del hombre al ser.

El pensamiento de Heidegger se desarrolla a partir de la discusión con dos corrientes filosóficas en boga durante las primeras décadas del siglo XX: el neokantismo, centrado en la reflexión acerca de la lógica, la teoría del conocimiento y de los valores, y el vitalismo de Nietzsche, Bergson y Dilthey. Heidegger manifiesta una clara preferencia por la filosofía de la vida, pues le parece que responde mejor a la crisis espiritual de su tiempo, cuya manifestación más evidente es la Primera Guerra Mundial. En el pensamiento de Heidegger incide, además, de manera decisiva su original formación religiosa, que lo hace ocuparse intensamente con el Nuevo Testamento, los Padres de la Iglesia, Lutero, y en general, la reflexión sobre la vida y la historicidad, que por lo demás, en los años previos a la Gran Guerra, eran omnipresentes en la literatura y la filosofía. Entre los años 1910 y 1914 se traducían al alemán las obras de Dostoyevsky y Kierkegaard, cobraba notoriedad la poesía de Rilke y Trakl, se editaban las Obras de Dilthey y se reeditaba la Voluntad de poder de Nietzsche. 
En este contexto se desarrolla también la filosofía fenomenológica, cuyo fundador, Edmund Husserl, se propone la misión de superar la crisis de la ciencia positivista que había dominado casi sin contrapeso la escena cultural europea durante gran parte de la segunda mitad del siglo XIX. El sentido de esta crisis consiste, para Husserl, en que la ciencia ha dejado de tener significado para el hombre, pues no le proporciona orientación sobre los problemas acuciantes del presente, ni criterios rigurosos para guiar su conducta. La fenomenología se presenta como una reflexión filosófica que quiere fundamentar firmemente la objetividad del saber mediante un método, cuya principal regla es dejar que "las cosas mismas" se hagan patentes en su contenido esencial, a través de una mirada intuitiva que haga presente las cosas tal como se dan inmediatamente para el que las vive y poniendo entre paréntesis el juicio sobre la validez de los presupuestos, opiniones o interpretaciones acerca de ellas.

La conciencia no es para la fenomenología algo cerrado en sí mismo, sino que está definida por la intencionalidad, es decir, por la particularidad de estar siempre abierta y referida a algo como su correlato objetivo. El análisis fenomenológico muestra, además, que los objetos no se dan a la conciencia aisladamente, sino insertos en un contexto mayor, en el que se destacan como lo que son. Este contexto es el mundo, horizonte general de todo lo que es y puede llegar a ser contenido de la experiencia. Husserl distingue dos formas de relación de la conciencia con el mundo. La primera es la forma natural o ingenua en que todos nos desenvolvemos en el mundo en el que nacemos, trabajamos, pensamos y morimos, y que por lo tanto se nos presenta como realmente existente. La segunda se refiere al mundo visto con la perspectiva del fenomenólogo, que ya no da por supuesta su existencia, sino que lo tematiza en forma crítica y reflexiva como constituido por actos intencionales de una conciencia pura. Pero es precisamente esa exigencia metodológica de la epojé, de poner entre paréntesis la existencia del mundo y de los sujetos y objetos reales para quedarse únicamente con la experiencia de la conciencia pura, analizable por un observador desinteresado, lo que Heidegger, asistente y discípulo de Husserl a partir de 1918, terminaría por rechazar para orientar la fenomenología hacia la hermenéutica.

\section{HEIDEGGER: LA TRANSFORMACIÓN DE LA FENOMENOLOGÍA}

Según Heidegger, el problema de la fenomenología, tal como la ha desarrollado su maestro, es que sucumbe a la enorme influencia que tiene la posición teórica en el pensamiento filosófico tradicional. Heidegger está de acuerdo con Husserl en relación al concepto de intencionalidad. Que la conciencia está directa e inmediatamente dirigida a su objeto, el cual nunca es un ingrediente de la propia conciencia, es, para Heidegger, el gran descubrimiento de las Investigaciones lógicas. Pero, a diferencia de Husserl, no considera que el flujo de las vivencias intencionales constituya un ámbito que deba ser investigado por sí mismo. Heidegger considera que Husserl permanece adherido al prejuicio moderno, que recibe a través de Brentano, de la existencia de una esfera subjetiva incuestionable. Ese sería un prejuicio que comparte con el psicologismo, a pesar de la dura crítica que le ha dirigido en las 
mismas Investigaciones lógicas (1). Heidegger considera que para combatir de verdad al psicologismo hay que criticar también la concepción de una esfera de la conciencia como región de investigaciones propias.

En el curso La idea de la filosofía y el problema de la cosmovisión, del semestre de invierno de 1919 (2) y en el curso sobre Problemas fundamentales de la fenomenología de 1919-20, Heidegger presenta una concepción de la fenomenología como ciencia originaria de la vida fáctica. En estos cursos Heidegger se refiere a la cientificidad de la filosofía como esencialmente ligada a su carácter originario, en el sentido de ser preteórico.

En esta época Heidegger considera que la filosofía es revivencia o repetición de la vida inmediata y espontánea. Pero esta repetición no puede tener la forma de la reflexión que propone Husserl, porque esta produce una modificación esencial en la vivencia primitiva. La reflexión puede sacar a luz todo lo que estaba presente en la vivencia, pero no puede revivirla. Mi vivir no se me da como un objeto puesto ante mí, ni menos como una forma inmanente a la conciencia, sino como un ocuparse de variadas formas con las cosas, que me involucra y afecta, pues me puede resultar bien o mal. La repetición tiene que tomar íntegramente todo lo que aparece en el vivir inmediato, especialmente los modos no teóricos de ese aparecer. Esto equivale a adoptar una posición estrictamente opuesta a la reducción fenomenológica o epojé de Husserl, pues implica coejecutar la posición de la actitud natural, sumirse en ella, no ponerla fuera de circulación.

En el semestre de invierno de 1919-20, Heidegger dicta un curso sobre Fundamentos de la mística medieval, al cual siguen, en el semestre de invierno de 192021, otro sobre Introducción a la fenomenología de la religión, y en el semestre de verano de 1921, otro sobre San Agustín y el neoplatonismo. El tema dominante de estos cursos es la precariedad del aparato conceptual metafísico para dar cuenta adecuadamente de la experiencia viva del cristiano. La insuficiencia principal de la metafísica para exponer la experiencia de la vida y de la historia consiste, según Heidegger, en privilegiar, desde el punto de vista teórico, la simple presencia, olvidando, entre otras cosas, que la experiencia cristiana originaria del tiempo y de la historia no está dirigida al presente, sino al futuro, como expectativa del acontecimiento de la segunda venida de Cristo.

Por otra parte, desde las lecciones universitarias de 1919, Heidegger comienza a conectar su peculiar concepción de la fenomenología con la Ética a Nicómaco de Aristóteles, y particularmente con los análisis sobre la frónesis para ilustrar formas de comportamiento humano que no se dejan reducir al ámbito de lo teórico. En las Interpretaciones fenomenológicas sobre Aristóteles (1921-22), Heidegger se refiere a la triple estructura del comportamiento, distinguiendo aquello hacia lo que se dirige, el sentido referencial (Bezugssinn), el cómo es ejercido el comportamiento, su sentido de ejecución (Vollzugssinn), y el respecto de qué, la referencia o sentido de contenido (Gehaltssinn). El sentido de ejecución tiene un significado especial,

(1) Cf. Prolegomena zur reinen Logik, Husserliana XVIII.

(2) Curso de un semestre conocido como Kriegsnotssemester, es decir, "semestre de emergencia por causa de la guerra", dirigido a alumnos que habían combatido en la recién concluida Guerra Mundial, y que tuvo una duración de solo tres meses entre fines de enero y mediados de abril. 
pues a diferencia de la nóesis husserliana, no subraya unilateralmente la forma teórica o cognoscitiva del acto. Vollzug es una expresión que indica de modo general la acción de vivir o el cómo se realiza la vida, sin reducirla a los actos noéticos, aunque tampoco excluyéndolos. El Vollzugssinn tiene una primacía sobre los demás en la medida que determina de qué modo concreto se realiza la referencia al objeto y cómo este se hace presente. Los objetos no tienen un sentido independiente del modo como nos relacionamos o nos comportamos hacia ellos.

Heidegger es crítico respecto de la pretensión husserliana de captar esencias de un modo inmediato. La apertura de un fenómeno solo es posible sobre la base de una precomprensión del mismo, la cual se revelará como inadecuada si conduce a distorsiones y encubrimientos y como adecuada si muestra ser fecunda en el sentido de tener la fuerza suficiente para abrir el fenómeno que se quiere comprender. En lugar de un criterio absoluto de evidencia hay que hacerse cargo de la radical finitud e historicidad de todo esfuerzo cognoscitivo. En este sentido, la fenomenología debe vincularse a la hermenéutica, lo que ocurre especialmente a partir de las lecciones sobre Ontología o Hermenéutica de la facticidad, de 1923.

\subsection{Hermenéutica de la facticidad}

En estas lecciones, Heidegger convierte explícitamente la cuestión ontológica del sentido del ser en la pregunta central de su filosofar. Que la vida fáctica procure comprenderse a sí misma de un modo preteórico, es una cierta forma de ser en una peculiar relación con todas las otras cosas que son, y al ocuparse de sí misma está presente esa comprensión de su propio ser. Pero Heidegger no solo comienza a identificar la filosofía fenomenológica con la ontología, sino también a esta última como "hermenéutica de la facticidad", y define su tarea como "interpretación que la facticidad hace de sí misma", o el darse a conocer la vida fáctica a sí misma (3). Heidegger sostiene que el movimiento hermenéutico de la autointerpretación está esencialmente determinado por el hecho de que la vida fáctica se da de un modo distorsionado, pues siempre está encubriéndose a sí mismo. Este encubrimiento es tan originario como la noticia que la vida originaria tiene de sí misma. No es un encubrimiento absoluto, sino una especie de desfiguración. Eso es lo que hace posible la comprensión. El problema de la hermenéutica consiste en encontrar una interpretación que disuelva este encubrimiento originario.

La hermenéutica de la facticidad comienza preguntándose por la situación hermenéutica a partir de la cual comprender el ser de la vida fáctica. Para Heidegger no se puede adoptar ninguna actitud, ni siquiera la pura reflexión teórica, que no implique un determinado modo de situarse al que corresponde un modo correlativo de aparecer. No existe la pura autotransparencia de la mirada teórica, un puro reflejo neutro de lo que es. La mirada fenomenológica tiene una estructura intencional determinada por la propia vida fáctica, en la que se asienta toda forma de mirar. La situación hermenéutica se define por un lugar desde donde se mira, una dirección hacia la que se mira y un horizonte hasta donde llega la mirada y dentro del que se mueve lo que ella aspira a ver.

(3) GA 63, § 3; Ontología. Hermenéutica de la facticidad, pp. 32-40. 
El hilo conductor de la mirada hermenéutica lo constituye lo que Heidegger llama "indicación formal" (formale Anzeige), concepto que usó mucho antes de Ser y tiempo, aunque las referencias temáticas son escasas (4). La indicación formal es un concepto que no tiene un contenido material. Su modo de remitir a algo no está determinado por un conjunto de rasgos ónticos de la cosa que designa. No es un concepto susceptible de ser llenado por la presencia del objeto mentado, mediante una intuición impletiva, según la terminología de Husserl. Pero tampoco significa vaciedad completa, sino una exigencia de ejecución. Tenemos que recorrer un camino para que la indicación formal se llene de sentido. Solo es inteligible en los comportamientos mediante los cuales se ejerce. En la indicación formal hay un llamado a ejercer el concepto como condición del proceso hermenéutico de interpretación o de explicitación del contenido posible. No se trata simplemente de un método que el filósofo adopta en forma libre. La vida fáctica impone este procedimiento desde sí misma, y no mediante un artificio metódico, como la epojé de Husserl. Por eso Heidegger reemplaza la epojé por la disposición afectiva, como la angustia, que rompe con toda objetivación.

\subsection{La pregunta por el sentido del ser}

En Ser y Tiempo Heidegger vuelve a plantear la pregunta por el sentido del ser, y sostiene que la filosofía no puede lanzarse a ciegas sobre el ser, olvidando las condiciones bajo las cuales su sentido es buscado y podría ser hallado. La metafísica occidental no se habría hecho cargo suficientemente de estas cuestiones y no habría sabido, a juicio de Heidegger, distinguir adecuadamente el ser respecto de determinadas formas de entidad o conceptualizaciones abstractas. La pregunta por el ser debe plantearse en toda su radicalidad y a partir de la situación de quien la formula, situación que determina no solo la pregunta, sino también la dirección de la respuesta (5).

¿Cómo se articula la fenomenología con la hermenéutica? El concepto heideggeriano de "fenomenología" se basa en una peculiar interpretación de los conceptos de "fenómeno" y de "logos". Por "fenómeno" (Phänomen) entiende algo que no es una mera manifestación (Erscheinung) en el sentido de algo que se anuncia sin mostrarse, como por ejemplo los síntomas de una enfermedad, sino más bien la patencia o revelación de lo que la cosa es en sí misma. Tampoco es "apariencia" (Schein), es decir, algo que se muestra como lo que no es. Pero tanto la manifestación como la apariencia se fundan, de diferente manera, en el fenómeno. Por su

(4) Las principales referencias se encuentran en el curso del 20-21 sobre Introducción a la fenomenología de la religión, y en el curso del 21-22 sobre Interpretaciones fenomenológicas de Aristóteles.

(5) Heidegger distingue en toda pregunta aquello 1) por lo que se pregunta (das Gefragte), 2) a qué o quién se pregunta (das Befragte), un ente determinado, 3) de qué se pregunta, es decir, lo preguntado (das Erfragte) o aquello que se espera encontrar al llegar la pregunta a su meta. Aplicado a la pregunta que nos ocupa, aquello por lo que se pregunta es el ser y lo que se espera encontrar es el sentido del ser. Pero ¿a qué ente hay que dirigir la pregunta? La respuesta de Heidegger es: la pregunta debe ser dirigida a aquel ente que está constitutivamente abierto al ser porque se preocupa de su propio ser. Este ente no es otro que el hombre, pero desde esta perspectiva no interesa como ente con tales o cuales características, sino en su condición de radical apertura al ser. Cf. Sein und Zeit, § 2 . 
parte, el "logos" es entendido como el hacer ver aquello de lo que se habla, el desencubrimiento de lo que estaba encubierto. Por lo tanto, la fenomenología es el método que consiste en "hacer ver desde sí mismo aquello que se muestra, y hacerlo ver tal como se muestra desde sí mismo" (6).

Heidegger no puede estar de acuerdo con la reducción histórica que plantea Husserl para el método fenomenológico. Según Husserl, debemos contemplar las cosas con una mirada neutral, sin una predisposición determinada. Hay que desconectar todas las concepciones filosóficas, teológicas, científicas, axiológicas que impiden describir los fenómenos tal como se manifietan desnudamente a la conciencia. Heidegger considera, por el contrario, que poner entre paréntesis la historia, solo sirve para encubrir los prejuicios operantes en toda descripción que se vale de conceptos. Todos los conceptos están saturados de tradición y de teoría, de manera que si se pretende ignorar aquello, operan de todas maneras de un modo inadvertido. Para tener una relación libre con la historia es necesario realizar una "destrucción" fenomenológica de la ontología. Con ello no se trata de realizar una operación meramente negativa, sino de posibilitar un acceso positivo al fenómeno, mediante el desmontaje de las tendencias negativas ocultas y el descubrimiento de posibilidades nuevas que permanecen latentes en el pensamiento anterior (7).

Heidegger no niega que la filosofía tenga que regirse por las cosas mismas. Todo lo contrario, hace suyo el lema husserliano: “¡a las cosas mismas!” Pero el fenómeno del que se ocupa primeramente la fenomenología está primeramente oculto, encubierto, obstruido. Ese fenómeno no es otro que el ser, y es ocultado o encubierto por todos los demás fenómenos que acaparan nuestra atención de un modo inmediato. Es decir, no es fenómeno en el sentido antes descrito, por lo que tiene que ser sacado a luz, dado que se encuentra en la raíz de todos los otros fenómenos que se encuentran en primer plano.

\subsection{Comprensión e interpretación}

Para sacar a luz el sentido del ser es necesario partir de la caracterización del ser del Dasein, en tanto que abierto a la comprensión del ser, apertura que Heidegger designa como posibilidad de ser o como proyecto libre. La función de la hermenéutica del Dasein consiste en explicitar ese Dasein, en primer lugar para destruir los diferentes estratos de esa ocultación, pero también para sacar a luz el Dasein tal como puede ser en sí mismo para sí mismo, es decir para que cada Dasein pueda ser propiamente sí mismo. En el cuidado del Dasein por su propio ser arraigan las preguntas fundamentales de la filosofía; por lo tanto la hermenéutica del Dasein retorna o repercute (schlägt zurück) sobre el Dasein mismo (8). Se podría añadir, por otra parte, que la existencia propia o auténtica del Dasein repercute, a su vez, sobre el planteamiento adecuado de las preguntas filosóficas.

¿Cuáles son esas posibilidades de ser que de las que se ocupa y preocupa la existencia humana? No son posiblilidades infinitas, sino que están doblemente de-

(6) Sein und Zeit, § 7, p.34.

(7) Sein und Zeit, § 6 .

(8) Sein und Zeit, § 7, p. 38. 
terminadas. En primer lugar, hay posibilidades que no hemos escogido, pero con las que nos encontramos y nos condicionan: el haber nacido en tal lugar, con tal sexo, bajo tales condiciones sociales, etc. Nos encontramos fácticamente arrojados en el mundo de tal o cual modo que no depende de nosotros, lo que determina nuestra disposición de ánimo o modo de encontrarnos. Otras posibilidades sí pueden ser escogidas libremente, pues están disponibles o abiertas a la proyección que hagamos de nuestra existencia. Estas últimas son las que definen la comprensión como modo de ser del Dasein desde el cual las cosas adquieren significado. En el mundo circundante, cotidiano de las cosas "a la mano", todas las cosas están relacionadas entre sí y tienen una función y un significado comprensible, que depende del modo como los hombres proyectan su existencia. Las cosas no tienen un significado en sí mismas, pues significan algo distinto para alguien que se proyecta como artesano, como deportista, como científico, etc. Cada una de estas proyecciones determina en vista de qué hacemos uso de las cosas.

Heidegger llama "interpretación" a un desarrollo ulterior de la comprensión, la que se apropia de lo comprendido, haciéndolo expreso o explícito. Una forma elemental de interpretación que se da en la vida cotidiana es designada por Heidegger "ver en torno" o "circunspección" (Umsicht), que ocurre, por ejemplo, cuando se interrumpe el curso normal de la actividad práctica. Un martillo no funciona bien, lo que provoca un preguntarse por el "en cuanto qué" o "cómo" del martillo o de la actividad que trato de realizar con él. Esta pregunta puede llegar a derivar en caso extremo hasta la teoría pura sobre la esencia de los instrumentos en general.

En el $\S 32$ de Ser y Tiempo, Heidegger afirma que lo que hace posible la apropiación es el sentido, al que define así: "Sentido es el horizonte del proyecto, estructurado por el haber-previo, la manera previa de ver y la manera de entender previa, horizonte desde el cual algo se hace comprensible en cuanto algo" (9). El sentido es aquello en base a lo cual podemos comprender algo en tanto que algo, aquello hacia lo que se proyecta la existencia humana y desde lo cual es posible comprender mejor a los entes, hacerse cargo de ellos como algo, determinado. Es una estructura del hombre en tanto que Dasein, no es algo que esté adherido a los entes, u oculto detrás de ellos, ni flotando en algún espacio ideal. En ese "hacia dónde" ya está dado por anticipado lo que se comprende, de tres maneras: Lo que se interpreta se posee ya de antemano, recortado o perfilado de una cierta manera y acompañado de una cierta conceptualización.

Si la interpretación de mueve dentro de lo ya comprendido y se nutre de ello, entonces se mueve en un círculo. Pero no se trata de un círculo vicioso, sino de un círculo hermenéutico. Es decir, no es un círculo que haya que eliminar del saber, como aspiran a hacerlo algunos historiógrafos positivistas, sino un círculo en el que hay que entrar de manera adecuada. Lejos de hacer caducar las cosas mismas, conlleva, según Heidegger, la exigencia de legitimar la interpretación desde las cosas mismas: la "primera, constante y última tarea [de la interpretación] consiste en no dejar que el haber previo, la manera previa de ver y la manera de entender previa le sean dados por simples ocurrencias y opiniones populares, sino en asegurarse el 
carácter científico del tema mediante la elaboración de esa estructura de prioridad a partir de las cosas mismas" (10).

Heidegger distingue el "en cuanto" o la interpretación hermenéutico-existencial del "en cuanto" apofántico. Aristóteles llamaba interpretación o hermenéutica a toda oración compuesta de nombre y verbo, e incluso a las partes mismas de la oración. Pero consideraba que la interpretación en sentido más propio es aquella oración que puede ser verdadera o falsa, es decir la oración apofántica, a diferencia de los deseos, súplicas, órdenes, etc. Aunque parte de la distinción aristotélica, Heidegger se niega a aceptar la supremacía del "en cuanto" apofántico (enunciativo) sobre el hermenéutico. Según Heidegger el "en cuanto" o interpretación apofántica considera a las cosas como algo que "está ahí", "ante la vista" (vorhanden) o presente ante una contemplación teórica, no algo "a la mano" (zuhanden). Esta interpretación apofántica realiza una nivelación de las cosas convertidas en objeto de contemplación, sacándolas del contexto en que se las usa, o abstrayendo de la situación concreta en que se está con ellas. De esta manara se ocupa de sus propiedades generales, que formula mediante proposiciones o juicios teóricos. En cambio, el "en cuanto" hermenéutico es un "en cuanto" situado, que no abstrae de la dinámica concreta de la situación. La apófansis se distingue, pues, de la hermenéutica como un caso límite de esta que implica una desmundanización (11).

Consecuentemente, Heidegger afirma que la verdad como adecuación es también una verdad derivada. En efecto, la verdad-adecuación consiste en una relación de tipo especial, que es la relación tal-como. Se trata de una relación en la que de algún modo debe cumplirse una igualdad. Esta relación de igualdad se realiza al hacerse igual el contenido ideal del juicio con la cosa conocida, es decir, al entrar en relación de concordancia ese contenido ideal con la cosa expresada. Para que merezca el calificativo de verdadera, esa relación tiene que ser comprobada de alguna manera. Pero la comprobación exige que la cosa o el ente esté descubierto, que el objeto se muestre como él mismo, en su entidad. No puede haber verificación sin un previo mostrarse de los entes. Por ello Heidegger rehabilita el sentido originario del concepto griego de la verdad como alétheia o desencubrimiento (12).

Con estas consideraciones, Heidegger pone a la filosofía en la disyuntiva de seguir el camino tradicional orientado al discurso científico-apofántico o bien orientarse hacia aquellas formas del discurso que abren a una situación y que por lo mismo conducen a una verdad distinta a la de la concepción tradicional. En último término, el modelo que inspira a Heidegger es bíblico: la Palabra ilumina una situación e interviene en ella transformándola, por lo que tiene que ser interpretada y aplicada de maneras siempre nuevas en la historia.

Aunque la obra posterior a Ser y Tiempo, especialmente a partir del llamado giro (Kehre) de 1929 abandona en gran medida la terminología fundamental de aquel libro, incluyendo la que se refiere directamente a la hermenéutica, por considerarla todavía demasiado ligada a un enfoque subjetivista propio de la modernidad, Heidegger mantiene, y aún radicaliza, el rechazo a considerar el lenguaje enunciati-

(10) Sein und Zeit, p. 153.

(11) Sein und Zeit, § 33.

(12) Sein und Zeit, § 44. 
vo como sede de la verdad. Una de las pocas referencias de la obra tardía a la hermenéutica se encuentra en una conversación con un profesor japonés en 1953-54, la que fue registrada en el volumen titulado Unterwegs zur Sprache (De camino al habla, 1959). Consultado por el profesor japonés acerca del sentido de la hermenéutica, Heidegger contesta que en Ser y Tiempo hermenéutica no significa el arte de la interpretación, ni la interpretación misma, sino "la tentativa de determinar lo que es la interpretación a partir de lo que es hermenéutico". Naturalmente, el japonés no queda conforme y pregunta a qué se refiere lo "hermenéutico", recibiendo la siguiente respuesta: "la cuestión es enigmática y tal vez ni siquiera se trate de una cuestión (Sache)" (13). No obstante, avanzada la conversación Heidegger vuelve sobre el sentido de lo hermenéutico y remite al término griego hermeneuein, conectándolo con el dios Hermes, mensajero divino, de manera que resulta la siguiente caracterización: "hermeneuein es aquel hacer presente que lleva al conocimiento en la medida que es capaz de prestar oído a un mensaje" (14). De esta manera Heidegger subraya la originaria relación de la hermenéutica con el lenguaje en tanto que portador de un mensaje. La filosofía hermenéutica de Gadamer se aferrará firmemente a esta concepción.

\section{GADAMER: LA CONSOLIDACIÓN DE LA FILOSOFÍA HERMENÉUTICA}

Gadamer fue alumno de Heidegger a partir de 1923 en Marburgo. Al igual que su maestro, no concibe la comprensión como un sistema de reglas orientadas a la recta comprensión de cierto tipo de fenómenos, sino como una reflexión sobre lo que acontece con el hombre cuando efectivamente comprende. Por otra parte, Gadamer se aparta de Heidegger al hacer de la reflexión sobre los fundamentos de las ciencias del espíritu un tema central de su filosofía, al que dedicó lecciones universitarias entre 1936 y 1959, las que desembocaron en Verdad y Método.

Esta obra arranca de hecho de la pregunta por la adecuada autocomprensión de las ciencias del espíritu frente a las ciencias de la naturaleza. Una de las principales inspiraciones al respecto la recibe del naturalista Herrmann Helmholtz, quien en un discurso de 1862 sobre la relación entre las ciencias naturales y las ciencias del espíritu, afirmaba que estas últimas no proceden, como aquellas, obteniendo leyes generales por un proceso de inducción a partir de material empírico, sino que recurren más bien a un cierto tacto psicológico vinculado a la memoria y a la autoridad (15). Gadamer sintoniza más con Helmholtz que con Dilthey o los neokantianos que hacia fines del siglo XIX y comienzos del siglo XX se ocuparon del mismo problema, puesto que comparte con él una actitud crítica frente a la preocupación metodologista centrada en el problema de la cientificidad de las ciencias del espíritu. Gadamer considera que la naturaleza de las ciencias del espíritu se puede entender mejor desde la tradición humanista de la formación que de la idea moderna de la ciencia.

(13) GA 12, p. 93; De camino al habla, p. 90.

(14) GA 12, p. 115; De camino al habla, p.110.

(15) GW I, pp. 20-22; VM I, pp. 44-46. 
En ese sentido su investigación se propone cubrir el dominio de la investigación de la verdad (sobre todo de las ciencias del espíritu, pero no únicamente de ellas), superando el extrañamiento del hombre respecto del mundo que produce la conciencia metódico-científica, lo que implica, a su juicio, el tratar de poner al descubierto las condiciones que hacen posible la autocomprensión del hombre en las distintas esferas de su experiencia de la verdad.

\subsection{Arte e interpretación}

Gadamer se pregunta cómo ha llegado a ocurrir que la tradición humanista que impulsó el nacimiento de las ciencias del espíritu se viera desplazada por el afán metodológico propio de la concepción cientificista de la verdad, y concluye que al respecto fue determinante el efecto que tuvo la reflexión kantiana sobre la belleza y el arte en la Crítica de la facultad de juzgar. En efecto, en esa obra el buen gusto y el sentido común, que antes de Kant estaban asociados al conocimiento de lo humano y del orden social, quedan relegados al ámbito de la subjetividad estética y disociados del conocimiento propiamente tal. En la medida que para Kant la validez del conocimiento está determinada por los criterios metodológicos impuestos por las ciencias de la naturaleza, la pretensión de verdad del conocimiento estético y de las ciencias del espíritu queda desacreditada, a menos que estas últimas se arrimen a aquellos criterios. Gadamer procura contrarrestar esta tendencia mostrando, en primer lugar, un camino de comprensión de la verdad del arte que supere la subjetivación que a su juicio provoca la reflexión kantiana y, a partir de allí, encontrar un modelo de interpretación que pueda valer primariamente para las ciencias del espíritu, pero extensivo a todos los ámbitos que cubre la experiencia humana de la verdad.

Según Gadamer la estética postkantiana, especialmente a partir de Schiller, espera del arte una elevación de las condiciones de vida humana. Pero tal cosa implica un proceso de formación, es decir, una elevación hacia la generalidad, hacia una experiencia común y vinculante que no puede esperarse de los genios artísticos en tanto que individuos aislados, o a lo más referidos a pequeñas comunidades disgregadas e inconexas entre sí (16). La comprensión de la obra de arte apunta, según Gadamer, a un sentido común, en el que se representa algo que trasciende las subjetividades individuales. La representación artística no es mera copia o imitación de la realidad, sino que es representación en el sentido del juego. Cada juego plantea tareas que deben ser cumplidas por los jugadores. El cumplimiento de estas tareas significa representar el juego. Lo que se representa no es la subjetividad de los jugadores sino las tareas impuestas por el juego, esto es, se representa el juego mismo. El jugador solo logra representarse a sí mismo como jugador jugando el juego, es decir, representándolo. Ahora bien, que el juego sea efectivamente representado para espectadores reales o virtuales no es de la esencia de todo juego, pero sí lo es para aquella forma peculiar de juego que es, según Gadamer, la obra de arte: "La representación del arte implica esencialmente que se realice para alguien, aunque de hecho no haya nadie que oiga o que vea" (17).

(16) GW I, p. 93 s.; VM I, pp. 128 s.

(17) GW I, p. 116; VM I, p. 154. 
Lo que se representa en la obra de arte es, según Gadamer, lo plenamente verdadero, el ser más auténtico o esencial de las cosas, que normalmente está oculto y sustraído a la visión de los hombres. La superioridad de lo representado en la obra de arte respecto de la realidad cotidiana radica en que, a diferencia de esta, se da un círculo cerrado de sentido, un conjunto de posibilidades resueltas, no hay la indefinición de expectativas deseadas o temidas que no pueden cumplirse todas por ser excluyentes entre sí. El ser de la obra de arte es cumplimiento, resolución, actividad que no apunta más allá de sí misma a un futuro incierto, sino que tiene su telos en sí misma. En este sentido, el representar está lejos de ser una mera multiplicidad de vivencias cambiantes cuyo objeto fuera una especie de molde vacío que los sujetos llenan de significado de un modo completamente arbitrario; por el contrario, implica siempre interpretar la realidad. En la representación artística se destacan obligadamente algunas cosas y se dejan otras de lado, se exageran rasgos, etc., lo que introduce necesariamente una desproporción entre algo y la interpretación que se hace de ese algo como algo.

Ahora bien, si la representación artística es interpretación, debe cumplir los requisitos generales de toda interpretación, que estudia la hermenéutica. En particular no puede aislarse del mundo al que pertenece, ignorando bajo qué condiciones se muestran las obras de arte. Como juego, la obra solo alcanza su ser pleno cuando se la juega cada vez, es decir, cuando es representada por parte de unos sujetos situados históricamente; pero a la vez es mucho más que los que los sujetos que representan saben acerca de ella y de sí mismos. La representación artística es, por lo tanto, actualización, el hacer presente a unos espectadores una obra que no puede permanecer fijada en el pasado. Es un desarrollo de la comprensión que se refiere a lo pasado como algo que tiene sentido para el hombre de hoy, una superación de las distancias temporales -y de las diferencias entre los sujetos- que no trata de anular esas distancias y diferencias, sino de hacerlas productivas.

\subsection{Mediación histórica}

Según Gadamer, la estética tiene que ser subsumida en la hermenéutica y esta, a su vez, debe estar inspirada en el modelo de la comprensión de la obra de arte. Dado que la obra de arte no es nunca solo pasado sino que supera la distancia temporal, cabe preguntarse de qué manera se hace cargo la hermenéutica de esa mediación histórica. A este respecto, Gadamer sigue la concepción hegeliana según la cual la esencia de la historia no consiste en la restitución del pasado, sino "en la mediación del pensamiento con la vida actual" (18). Pero cuando se trata de precisar el cómo de esa mediación no sigue a Hegel, sino la descripción que vimos en Heidegger del desarrollo de la comprensión como estando continuamente determinada por un triple movimiento de anticipación: una manera previa de tener, una manera previa de ver y una manera previa de concebir. Gadamer lo formula así: "El que quiere comprender un texto realiza siempre un proyectar. Tan pronto como aparece en el texto un primer sentido, el intérprete proyecta enseguida un sentido del todo. Naturalmente que el sentido solo se manifiesta porque ya uno lee el texto desde determinadas expectativas relacionadas

(18) GW I, p. 174; VM I, p. 222 
a su vez con algún sentido determinado. La comprensión de lo que pone en el texto consiste precisamente en la elaboración de este proyecto previo, que por supuesto tiene que ir siendo constantemente revisado en base a lo que vaya resultando conforme se avanza en la penetración del sentido" (19).

La interpretación se basa, entonces, en preconcepciones que van siendo corregidas a medida que se comprueba su falta de adecuación a las cosas mismas. El que las opiniones previas y los prejuicios tengan que ser examinadas en su pretensión de validez no significa que tengan que ser rechazados de antemano, pues su propio concepto admite la doble posibilidad de que su contenido sea verdadero falso. Gadamer no solo se enfrenta a la devaluación del prejuicio, sino también a la consecuente devaluación de la autoridad y de la tradición, consideradas por la Ilustración como expresiones de irracionalidad. Gadamer retruca, en cambio, que tanto el reconocimiento de una autoridad auténtica como la decisión de conservar determinadas tradiciones constituyen actos de la razón.

El concepto de tradición - una forma de autoridad consagrada por el pasado, que se ha hecho anónima y que determina nuestra acción, comportamiento e instituciones (20) - es fundamental para la comprensión de la mediación histórica que propone Gadamer, pues a partir de él describe la comprensión como interpenetración del movimiento de la tradición y del movimiento del intérprete. La tarea de la hermenéutica se basa, a su juicio, en el punto medio entre familiaridad y extrañeza que significa la tradición. En tanto que pertenecemos a las tradiciones estamos familiarizados con ellas; pero en tanto que estamos distanciados históricamente con sus fuentes nos resulta extraña y distante. " $Y$ este punto medio es el verdadero topos de la hermenéutica" (21).

Gadamer considera que la tradición y la investigación histórica forman un "entramado de efectos recíprocos", al que llama "wirkungsgeschichtliches Bewusstsein", "conciencia histórico-efectual", o mejor, "conciencia de los efectos de la historia". Esta unidad de efectos recíprocos entre la tradición, que viene del pasado, y la investigación histórica, que transcurre en el presente, tiene, a su juicio, algunos presupuestos. En primer lugar, hay una circularidad de la relación entre sujeto y objeto en la que ninguno de los dos polos de la relación es determinante por sí solo, pues en el acontecer de la comprensión el sujeto se forma como tal en la misma medida que es parte del proceso objetivo de la historia (22).

En segundo lugar, este enfoque presupone lo que Gadamer denomina "anticipación de la perfección" (23). Lo primero que importa cuando se lee un texto es comprender el asunto, lo que lleva a presuponer que el texto lo expresa en forma perfectamente adecuada y verdadera. Solo secundariamente, al advertir que este presupuesto es insostenible, se trata de "comprender" el texto como la opinión de otro, que debe ser corregida.

En tercer lugar, la distancia temporal entre el presente del intérprete y el pasado del que proviene la tradición no es simplemente un obstáculo para la compren-

(19) GW I, p. 271; VM I, p. 333.

(20) GW I, p. 286 s.; VM I, pp.348 s.

(21) GW I, p. 300; VM I, pp. 365.

(22) GW I, p. 298 s.; VM I, p. 363.

(23) GW I, p. 299 s.; VM I, pp. 363 s. 
sión, sino que tiene un rendimiento productivo para ella, no meramente porque permita desconectar en alguna medida los intereses del intérprete sobre el objeto, sino sobre todo porque esa distancia permite apreciar mejor los efectos de la obra en las distintas épocas: lo que perdura y lo que pierde validez, los prejuicios verdaderos y los prejuicios injustificados que la acompañan (24).

Ahora bien, Gadamer destaca que poner en suspenso los prejuicios para examinar sus pretensiones de validez es lo que define la pregunta, en tanto que sitúa nuestras opiniones en el terreno de lo abierto. Lo trasmitido nos interpela, poniendo en cuestión nuestras convicciones actuales. Pero para poder responder a ese cuestionamiento, nosotros, los interpelados, debemos, a su vez, plantear ciertas preguntas al pasado, interrogando a los textos que ese pasado nos lega (25).

En este cuestionamiento recíproco del presente y del pasado ocurre lo que Gadamer llama la fusión de horizontes. Poniendo en juego los prejuicios es posible destacar aspectos del pasado que son significativos para el intérprete, los que a su vez permiten destacar aspectos del presente que abren perspectivas de análisis del pasado. El horizonte del presente no se forma al margen del pasado, pero el horizonte del pasado también está sometido a los efectos del presente: "Comprender es siempre el proceso de fusión de estos presuntos 'horizontes para sí mismos'. [...] La fusión tiene lugar constantemente en el dominio de la tradición, pues en ella lo viejo y lo nuevo crecen siempre juntos hacia una validez llena de vida, sin que lo uno ni lo otro lleguen a destacarse explícitamente por sí mismos" (26).

En este proceso de la fusión de horizontes entra en juego el problema hermenéutico central, que a juicio de Gadamer es el problema de la aplicación, tradicionalmente reducido a un aspecto específico de las hermenéuticas teológica y jurídica. Gadamer considera, en cambio, que estas hermenéuticas son ejemplos paradigmáticos de algo que pertenece constitutivamente a toda auténtica comprensión. Siguiendo el modelo aristotélico de la frónesis, sostiene que la aplicación en sentido hermenéutico no presupone un concepto o ley general bajo el cual se subsume un caso particular, sino al revés, la concreción del caso particular es la que hace posible la auténtica comprensión de lo general.: “[...] si el texto, ley o mensaje de salvación, ha de ser entendido adecuadamente, esto es, de acuerdo con las pretensiones que él mismo mantiene, debe ser comprendido en cada momento y en cada situación concreta de una manera nueva y distinta. Comprender es siempre también aplicar" (27).

\subsection{Lenguaje y filosofía}

La tercera parte de Verdad y Método está basada en la tesis de que "el lenguaje es el medio universal en el que se realiza la comprensión" (28). En su lucha contra el distanciamiento metodológico, la hermenéutica gadameriana parte del supuesto que toda incomprensión, malentendido o extrañeza supone y está precedida por una comprensión o acuerdo que la sostiene. Pero la naturaleza de este acuerdo es esen-

(24) GW I, pp. 302 ss.; VM I; pp. 367 ss.

(25) GW I, pp. 375-481; VM I; pp. 447-454.

(26) GW I, pp. 311 s.; VM I; pp. 376 s.

(27) GW I, p. 314; VM I; p. 380.

(28) GW I, p. 392; VM I; p. 467. 
cialmente lingüística y se refiere al mundo que se representa en la vida común (29). El lenguaje es el elemento que permite superar la experiencia puramente regional o parcial, que implica extrañeza frente a otras experiencias igualmente parciales. No hay afirmación que no pueda ser comprendida como respuesta a una pregunta, es decir, en el marco de la interpretación de los mensajes en los que se realiza y comprende a la vez el ser del hombre como finitud abierta a una infinitud de sentido. "Todo hablar humano es finito en el sentido de que en él yace la infinitud de un sentido por desplegar e interpretar. Por eso tampoco el fenómeno hermenéutico puede ilustrarse si no es desde esta constitución fundamentalmente finita del ser, que desde sus cimientos está construida lingüísticamente" (30).

La hermenéutica de Gadamer considera el lenguaje como una realidad cargada con un significado ontológico, pues el ser acontece en el lenguaje como verdad, como desvelamiento de sentido que no es esencialmente distinto a las diferentes representaciones finitas en las que accede a la subjetividad humana. Gadamer sigue a Heidegger en su caracterización de la verdad como alétheia, desocultamiento del ser, y comparte plenamente su crítica al dominio del logos apofántico en la tradición filosófica occidental. Pero la salida de este dominio tiene en Gadamer una inflexión nueva respecto de Heidegger, pues no apunta solo a iluminar el trato práctico del hombre con las cosas en el mundo cotidiano, sino sobre todo a recordar que todo enunciado brota de un contexto dialógico a partir del cual únicamente puede extraer su sentido. Gadamer sostiene que el lenguaje solo realiza su verdadero ser en la conversación, en el ejercicio del entendimiento mutuo, mediante el cual pueden ser abordados y eventualmente resueltos los malentendidos (31).

Según Gadamer, gracias al lenguaje el fenómeno hermenéutico adquiere un alcance universal: no solo los fenómenos histórico-espirituales, sino todo cuanto puede ser comprendido es, en principio, comprensible, justamente porque puede ser articulado lingüísticamente (aunque de hecho no lo sea). Sin la posibilidad de la representación lingüística no tendría sentido pretender que verdaderamente se comprende algo, y no hay nada acerca de lo cual no pueda decirse alguna cosa con sentido. Sin palabras que la puedan expresar, la comprensión queda trunca. Este es el sentido de la afirmación, muchas veces mal entendida, según la cual: "El ser que puede ser comprendido es lenguaje" (32).

Pero al mismo tiempo las palabras que efectivamente pronunciamos no logran expresar adecuadamente lo que tenemos en mente al comprender. Gadamer recoge la

(29) GW I, p. 450; VM I; p. 535. Desarrollos ulteriores de la hermenéutica critican la idea de que exista un acuerdo previo o un consenso previo constitutivo del ser del hombre. Habermas, p.ej., llama la atención sobre ciertas distorsiones sistemáticas del lenguaje, de tipo ideológico, motivadas por factores de poder que rompen con la comunicación, y que por lo tanto no podrían ser resueltos simplemente retrocediendo a esa supuesta esfera común, sino que exigirían una metahermenéutica, una crítica de las ideologías que estableciera las condiciones para un acuerdo posible bajo condiciones ideales de comunicación, es decir, una comunicación libre de coacciones. Cf. Habermas: La lógica de las ciencias sociales, pp. 277 ss. Un intento de mediación entre ambas posiciones se encuentra en Paul Ricoeur: "Hermenéutica y crítica de las ideologías". En: Del texto a la acción, pp. 307-347.

(30) GW I, p. 462; VM I, p. 549

(31) GW I, pp. 372-384, 449 s.; VM I, p. 444-458, 535; cf. Tb. GW II, pp. 151 ss.; VM II, pp. 150ss.

(32) GW I, p. 478; VM I, pp. 567 s. 
doctrina agustiniana del verbo interior (33), para explicar, entre otras cosas, de dónde nace la necesidad de filosofar. Dado que las palabras no pueden dar adecuada cuenta de todo lo que se comprende cuando algo se comprende y de todo lo que se dice cuando algo se dice, ellas siempre se quedan cortas y llaman a seguir buscando más palabras que permitan desarrollar la comprensión, es decir, interpretar, y finalmente hacer filosofía (34). No filosofamos porque estemos en posesión de la verdad absoluta, sino justamente porque ella nos falta

\section{BIBLIOGRAFÍA}

Gadamer, Hans-Georg: Gesammelte Werke (GW). Mohr: Tübingen.

- Vol. I: Hermeneutik I: Wahrheit und Methode. Grundzüge einer philosophischen Hermeneutik, 1990 (6a ed.).

- Vol. II: Hermeneutik II: Wahrheit und Methode. Ergänzungen, Register, 1993 (2 ed.).

- Vol. III: Neuere Philosophie I: Hegel-Husserl-Heidegger, 1987.

- Vol. X: Hermeneutik im Rückblick, 1995.

Traducciones al castellano:

Verdad y Método (VM). Sígueme: Salamanca.

- Vol I. Trad. de A. A. Aparicio y R. de Agapito, 1977.

- Vol II. Trad. de M. Olasagasti, 1992.

Gadamer, Hans-Georg y Boehm, Gottfried (editores): Seminar: Philosophische Hermeneutik. Suhrkamp: Frankfurt a. M. 1979.

Grondin, Jean: Einführung in die philosophische Hermeneutik. Wissenschaftliche Buchgesellschaft: Darmstadt 1991.

Der Sinn für Hermeneutik. Wissenschaftliche Buchgesellschaft: Darmstadt 1994.

Habermas, Jürgen: Zur Logik der Sozialwissenschaften. Suhrkamp: Frankfurt a. M. 1985.

Traducción al castellano:

La lógica de las ciencias sociales. Trad. por M. Jiménez Redondo. Tecnos: Madrid 1988.

Heidegger, Martin: Sein und Zeit. Max Niemeyer: Tübingen 1986 (16 a ed.)

Gesamtausgabe (GA). Klostermann: Frankfurt a. M.

- Vol. 12: Unterwegs zur Sprache. Ed. por F.-W. von Herrmann. Klostermann: Frankfurt a. M. 1985.

- Vol. 56/57: Zur Bestimmung der Philosophie (1919) (GA 56/57). Ed. por B. Heimbüchel. Klostermann: Frankfurt a. M 1987.

- Vol. 58: Grundprobleme der Phänomenologie (1919-20). Ed. por: H.-H. Gander. Klostermann: Frankfurt a. M. 1993.

- Vol. 60: Phänomenologie des religiösen Lebens. Ed. por M. Jung, Th. Regehly, C. Strube. Klostermann: Frankfurt a. M 1995.

(33) GW I, pp. 424 ss.; VM I; pp. 504 ss.

(34) GW I, p. 474; VM I; p. 564. 
- Vol. 61: Phänomenologische Interpretationen zu Aristoteles. Einführung in die phänomenologische Forschung. Ed. por W. Bröker y K. Bröker-Oltmanns. Klostermann: Frankfurt a. M. 1985.

Traducciones al castellano:

Ser y Tiempo. Trad. de J. E. Rivera. Editorial Universitaria: Santiago de Chile, 1997.

Ontología. Hermenéutica de la facticidad. Versión de J. Aspiunza. Alianza: Madrid 1999.

Los problemas fundamentales de la fenomenología. Trad. y Prólogo de J. J. García Norro.Trotta: Madrid 2000.

De camino al habla. Versión de Y. Zimmermann. Ediciones del Serbal: Barcelona 1990 (2a ed).

Interpretaciones fenomenológicas sobre Aristóteles. Indicación de la situación hermenéutica. [Informe Natorp]. Edición y traducción de J. A. Escudero. Trotta: Madrid 2002.

Husserl, Edmund: Gesammelte Werke (Husserliana). Martinus Nijhoff: Den Haag.

- Vol. XVIII-XIX/1-2: Logische Untersuchungen Ed. por E. Holenstein y U. Panzer, 1975-1984.

- Vol. III 1/2: Ideen zu einer reinen Phänomenologie und phänomenologischen Philosophie. Erstes Buch Ed. Por K. Schuhmann, 1977 (2a ed.).

- Vol. VI: Die Krisis der europäischen Wissenschaften und die transzendentale Phänomenologie. Ed. por W. Biemel, 1962 (2a ed.).

Traducciones al castellano:

Investigaciones lógicas. Trad. de J. Gaos y M. García Morente. Revista de Occidente: Madrid 1967.

Ideas relativas a una fenomenología pura y una filosofía fenomenológica. Traducción de J. Gaos. Fondo de Cultura Económica: México 1949.

Crisis de las ciencias europeas y la fenomenología trascendental. Trad. por H. Steinberg. Folios: México 1984.

Kisiel, Theodore: The Genesis of Heidegger's Being and Time. University of California Press: Berkeley 1983.

Pöggeler, Otto: El camino del pensar de Martin Heidegger. Trad. de F. Duque. Alianza: Madrid 1993, $2^{\text {a }}$ ed.

Pöggeler, Otto: Hermeneutische Philosophie. Nymphenburger Verlagshandlung: München 1972.

Ricoeur, Paul: Du texte à l'action. Essays de hermeneutique II. Éditions du Seuil: Paris 1986.

Traducción al castellano: Del texto a la acción. Ensayos de hermenéutica II. Trad. por P. Corona. Fondo de Cultura Económica Argentina: Buenos Aires 2001.

Rodríguez, Ramón: La transformación hermenéutica de la fenomenología. Una interpretación de la obra temprana de Heidegger. Tecnos: Madrid 1997. 


\title{
RESUMEN
}

En primer lugar se expone sucintamente cómo Martin Heidegger, desde los inicios de su enseñanza universitaria, transforma la fenomenología en una filosofía hermenéutica, haciendo hincapié en los conceptos de comprensión e interpretación que ofrece su obra Ser y Tiempo. En segundo lugar se examinan algunos rasgos fundamentales de la filosofía hermenéutica, con especial referencia al arte, la historia y el lenguaje, tal como se presentan en la obra que consolida y consagra esta vertiente de la filosofía contemporánea: Verdad y Método de HansGeorg Gadamer.

\begin{abstract}
In this article the author first discusses the way in which Martin Hiedegger, form his early days of university teaching, turned the phenomenology into a hermeneutic philosophy placing special emphasis on the concepts of comprehension and interpretation of his work Being and Time. Then, the article goes on to examine some of the most fundamental aspects of the hermeneutic philosophy, paying especial attention to the arts, history and language, as presented in his work, which comes to consolidate this contemporary philosophical current: Truth and Method by Hans-Georg Gadamer
\end{abstract}

